\title{
BTZ black holes inspired by noncommutative geometry
}

\author{
Farook Rahaman* \\ Department of Mathematics, Jadavpur University, Kolkata 700 032, West Bengal, India
}

P.K.F. Kuhfittig才

Department of Mathematics, Milwaukee School of Engineering, Milwaukee, Wisconsin 53202-3109, USA

B.C. Bhu团 and Masiur Rahaman

Department of Mathematics, Meghnad Saha Institute of Technology, Kolkata-700150, India

\author{
Saibal Ray \\ Department of Physics, Government College of Engineering and Ceramic Technology, Kolkata 700 010, West Bengal, India \\ U.F. Monda承 \\ Department of Mathematics, Behala College, Parnasree, Kolkata 700060, India
}

(Dated: October 1, 2018)

\begin{abstract}
In this paper a Bañados, Teitelboim and Zanelli (BTZ) [1] black hole is constructed from an exact solution of the Einstein field equations in a $(2+1)$-dimensional anti-de Sitter spacetime in the context of noncommutative geometry. The BTZ black hole turns out to have two horizons, no horizon or a single horizon corresponding to a minimal mass. Certain thermodynamical properties are investigated, including Hawking temperature, entropy and heat capacity. Also discussed is the geodesic structure of BTZ black holes for both massless and massive particles. In particular, it is shown that bound orbits for test particles are possible.
\end{abstract}

PACS numbers: 04.40.Nr, 04.20.Jb, 04.20.Dw

\section{INTRODUCTION}

Recent years have seen rapid advances in the applications of noncommutative geometry, an outgrowth of string theory. The approach is based on the realization that coordinates may become noncommuting operators on a $D$ brane [2 5]. The result is a discretization of spacetime due to the commutator $\left[\mathbf{x}^{\mu}, \mathbf{x}^{\nu}\right]=i \theta^{\mu \nu}$, where $\theta^{\mu \nu}$ is an antisymmetric matrix. It is similar to the way that the Planck constant $\hbar$ discretizes phase space [2]. The noncommutativity eliminates point-like structures and replaces them with smeared objects. The noncommutative geometry is an intrinsic property of spacetime and does not depend on particular features such as curvature.

A number of studies inspired by noncommutative geometry can be found in the literature. In one of the earlier studies, Garattini and Lobo [6] obtained exact wormhole solutions that were then analyzed in semiclassical gravity. In a subsequent study [7] they found an exact gravastar solution and explored its physical characteristics. Rahaman et al. [8], discussing galactic rotation curves, concluded that a noncommutativegeometry background is sufficient for producing stable

\footnotetext{
*Electronic address: rahaman@iucaa.ernet.in

$\dagger$ Electronic address: kuhfitti@msoe.edu

${ }^{\ddagger}$ Electronic address: bikas`bhui@rediffmail.com

$\S$ Electronic address: mosiurju@gmail.com

ฯElectronic address: saibal@iucaa.ernet.in

**Electronic address: umarfarooquemondal@ymail.com
}

circular orbits without the need for dark matter. Kuhfittig [9] found that a special class of thin-shell wormholes that are unstable in classical general relativity exhibit small regions of stability in noncommutative geometry. In another study by Rahaman et al. [10] on higherdimensional wormholes, it is shown that wormhole solutions exist in the usual four, as well as in five dimensions, but they do not exist in higher-dimensional spacetimes. In a more recent study, Radinschi et al. 11] calculated the energy-momentum for a noncommuting radiating Schwarzschild black hole in order to obtain the expression for energy. Common to all these studies is that the effect of the smearing is mathematically implemented by using a Gaussian distribution of minimal length $\sqrt{\theta}$ instead of the Dirac delta function.

Interest in $(2+1)$-dimensional gravity has increased in recent years due to the discovery of various aspects of black-hole solutions. Some general works in this line are: quasinormal modes of charged dilaton black holes in $(2+1)$-dimensional solutions in low-energy string theory with asymptotically anti-de Sitter spacetimes [12], branes with naked singularities, analogous to linear or planar defects in crystals and showing that zero-branes in AdS spacetimes are "negative mass black holes" [13], Hawking radiation from covariant anomalies in $(2+1)$ dimensional black holes [14] and so on. On the other hand, specialized investigations have been carried out by Rahaman et al. [15] on gravastars in $(2+1)$ anti-de Sitter spacetimes with charge as an alternative to charged black holes. Also, nonstatic charged BTZ-like black holes in $(N+1)$-dimensions have been considered by Ghosh 
[16], which in the static limit, for $N=2$, reduces to $(2+1)$ BTZ black hole solutions. There are also charged, regular extensions of the BTZ black hole solutions available in the literature by employing nonlinear Born-Infeld electrodynamics to eliminate the inner singularity [17].

There are several proposals in the literature for constructing noncommutative black holes [18 21]. However, following Nicolini et al [3], we construct a BTZ black-hole solution from the Einstein field equations in $(2+1)$-dimensional anti-de Sitter spacetime, given a noncommutative-geometry background. This is followed by a discussion of the black hole's thermodynamical properties such as Hawking temperature, entropy and heat capacity, as well as the geodesic structure for both massless and massive particles. Some of the thermodynamical properties are similar to those obtained by Liang and Liu 22] who used a Lorentzian smeared mass distribution instead of a Gaussian one in $A d S_{3}$ spacetime. One consequence of this is that in the limit, $r / \sqrt{\theta} \rightarrow \infty$, the solution reduces to a rotating BTZ black hole. Returning to a Gaussian framework, rotating black holes in $(2+1)$ dimensions are also discussed in Ref. [23] by Tejeiro and Larranaga. They [24] also describe charged black holes and compare them to charged BTZ black holes.

\section{THE INTERIOR SPACETIME}

Let us write the line element describing the interior spacetime of a static spherically symmetric distribution of matter in $(2+1)$ dimensions in the following form:

$$
d s^{2}=-f(r) d t^{2}+[f(r)]^{-1} d r^{2}+r^{2} d \phi^{2},
$$

where $f(r)$ is denoted by $e^{2 \nu(r)}$ and $[f(r)]^{-1}$ by $e^{2 \mu(r)}$. We take the matter distribution to be anisotropic in nature and therefore choose the most general energymomentum tensor in the form

$$
T_{i j}=\left(\rho+p_{t}\right) u_{i} u_{j}+p_{t} g_{i j}+\left(p_{r}-p_{t}\right) \chi_{i} \chi_{j},
$$

where $\rho, p_{r}$ and $p_{t}$ represent the energy density, radial pressure and tangential pressure, respectively. Also, $\chi^{i}=$ $e^{-\mu(r)} \delta_{r}^{i}$ is a unit four vector along the radial direction and $u^{i}$ is the 4-velocity of the fluid.

The Einstein field equations with cosmological constant $(\Lambda<0)$, together with the general energymomentum tensor given in Eq. (2), yield (letting $G=$ $c=1$ )

$$
\begin{aligned}
2 \pi \rho+\Lambda & =\frac{\mu^{\prime} e^{-2 \mu}}{r}, \\
2 \pi p_{r}-\Lambda & =\frac{\nu^{\prime} e^{-2 \mu}}{r}, \\
2 \pi p_{t}-\Lambda & =e^{-2 \mu}\left(\nu^{\prime 2}+\nu^{\prime \prime}-\nu^{\prime} \mu^{\prime}\right) .
\end{aligned}
$$

We have, in addition, the conservation equation in $(2+1)$ dimensions:

$$
\left(\rho+p_{r}\right) \nu^{\prime}+p_{r}^{\prime}+\frac{1}{r}\left(p_{r}-p_{t}\right)=0 .
$$

In $(2+1)$ dimensions, the maximally localized source of energy of the static and spherically symmetric distributions having a minimal spread Gaussian profile is taken as [4]

$$
\rho=\frac{M}{4 \pi \theta} \exp \left(-\frac{r^{2}}{4 \theta}\right) .
$$

Here $M$ is the total mass of the source. Due to the uncertainty, it is diffused throughout a region of linear dimension $\sqrt{\theta}$.

The vacuum Einstein field equations in $(2+1)$ spacetime dimensions, with a negative cosmological constant, admit a black hole solution known as a BTZ solution [1]. For a BTZ black hole, we have $g_{r r}=g_{t t}^{-1}$. So to retain the structure, we require that

$$
p_{r}=-\rho
$$

This ansatz is known in the literature as a ' $\rho$-vacuum' or 'vacuum equation of state' in connection with the 'zero point energy of quantum fluctuation' [25 28] where pressure is of a repulsive nature.

With this equation, one can solve Eq. (6) to yield

$$
p_{t}=\frac{M\left(\frac{r^{2}}{2 \theta}-1\right)}{4 \pi \theta} \exp \left(-\frac{r^{2}}{4 \theta}\right) .
$$

Using the field equations, we get the following solution for the metric coefficients:

$$
e^{-2 \mu}=e^{2 \nu}=-A+2 M \exp \left(-\frac{r^{2}}{4 \theta}\right)-\Lambda r^{2},
$$

where $A$ is an integration constant.

In the limit, $\frac{r}{\sqrt{\theta}} \rightarrow \infty$, so that Eq. (10) reduces to a BTZ black hole, where the integration constant $A$ plays the role of the mass of the BTZ black hole, i.e., $A=M$. Observe that asymptotically far away, $\rho=p_{r}=p_{t}=0$.

To determine the mass distribution from Eq. (77), we use an approach similar to that in Refs. [4] and [6]:

$$
m(r)=\frac{M}{\pi^{(\tilde{m}-2) / 2}} \gamma\left[\frac{\tilde{m}}{2}, \alpha^{2}\left(\frac{r}{2 M}\right)^{2}\right],
$$

where $\alpha^{2}=M^{2} / \theta$ and $\gamma$ is the lower incomplete gamma function

$$
\gamma\left(\frac{a}{b}, x\right)=\int_{0}^{x} u^{a / b} e^{-u} \frac{d u}{u}
$$

For a BTZ black hole, $\tilde{m}=2$, and we obtain from $\alpha^{2} r^{2} / 4 M^{2}=r^{2} / 4 \theta$ the expression for mass as

$$
m(r)=M \int_{0}^{r^{2} / 4 \theta} e^{-t} d t=M\left[1-\exp \left(-\frac{r^{2}}{4 \theta}\right)\right]
$$

The parameter $\alpha$ plays a critical role in determining the horizons, as we will see later on. At the origin, $m(0)=0$, which is consistent with Eq. (11). 
Near the origin, the geometry is given by

$$
e^{-2 \mu}=e^{2 \nu}=-A+2 M-\left(\frac{2 M}{4 \theta}+\Lambda\right) r^{2}+\mathcal{O}\left(r^{4}\right) .
$$

One can identify this result with a BTZ black hole spacetime, where the total mass $M$ and the noncommutative parameter $\theta$ combine to modify the cosmological constant, a point also made in Ref. [4]. This indicates that different mass particles experience different cosmological constants. We therefore conclude that our line element describes the geometry of a noncommutative-geometry inspired BTZ black hole.

\section{FEATURES OF THE BLACK HOLE}

In this section we study some of the effects of the noncommutative geometry on BTZ black holes. Let $A=M$ in Eq. (10). Then the equation $g_{t t}\left(r_{h}\right)=0$ gives the event horizon(s):

$$
r_{h}^{2}=\frac{M}{\Lambda}\left[2 \exp \left(-\frac{r_{h}^{2}}{4 \theta}\right)-1\right] .
$$

Even though we cannot obtain a closed-form solution for $r_{h}$ in Eq. (14), we can readily write the mass $M$ as a function of $r_{h}$ :

$$
M=\frac{\Lambda r_{h}^{2}}{2 \exp \left(-\frac{r_{h}^{2}}{4 \theta}\right)-1} .
$$

The existence of horizons and their radii can be found at the points where $g_{t t}$ cuts the $r$-axis, as shown in Fig. 1 , using $\Lambda \sqrt{\theta}=-0.02$. Here three possibilities present themselves graphically in terms of the approximate value of $\alpha$ :

(i) two horizons when $\alpha>0.214$, or $M>M_{0}=0.214 \sqrt{\theta}$;

(ii) one horizon corresponding to the extremal black hole with $M=M_{0}$, i.e., $\alpha=0.214$;

(iii) no horizon for $\alpha<0.214$.

Fig. 1 shows that a noncommutative-geometry inspired BTZ black hole has two horizons and that the distance between the horizons will increase with an increasing black-hole mass. Fig. 1 also indicates that there is a minimal mass $M_{0}$ below which no black hole exists. Moreover, at the minimal mass $M=M_{0}$, the two horizons coincide at the minimal horizon radius $r_{0}$, which lies between the horizons. This $r_{0}$ is therefore the horizon radius of the extremal black hole. It can also be determined from the conditions $f=0$ and $\frac{d f}{d r}=0$, leading to the equation

$$
2 \exp \left(\frac{r_{0}^{2}}{4 \theta}\right)+\frac{r_{0}^{2} / \theta}{1-2 \exp \left(-\frac{r_{0}^{2}}{4 \theta}\right)}=0 .
$$

Using the condition $\Lambda \sqrt{\theta}=-0.02$ in Fig. 1, we can obtain $r_{0} / \sqrt{\theta}=2.59$, showing that the two approaches are consistent.

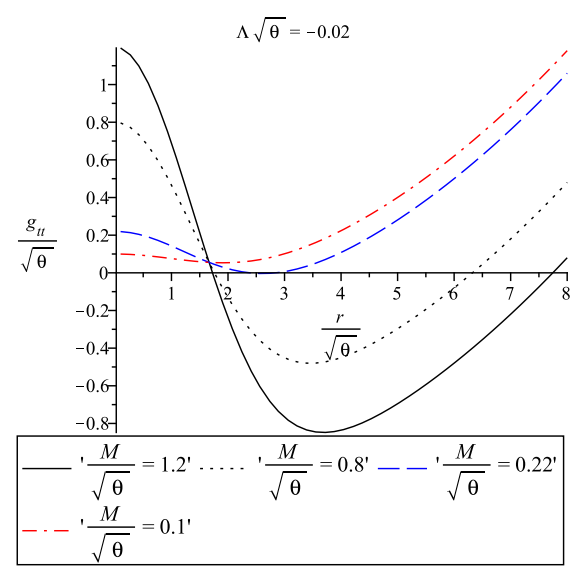

FIG. 1: The singularities occur where $g_{t t}$ cuts $r$-axis. Representation: the solid curve for $M=1.2 \sqrt{\theta}$ and dotted curve for $M=0.8 \sqrt{\theta}$ indicate two horizons. The dashed curve for $M=0.214 \sqrt{\theta}$ represents one degenerate horizon $r_{0} \approx 2.59 \sqrt{\theta}$, i.e., an extremal black hole. For $M=0.1 \sqrt{\theta}$, no horizon exists (chain curve). The intercepts on the $r$-axis give the radii of the event horizons.

The minimal mass of the extremal black hole can be written in terms of the minimal radius $r_{0}$, so that

$$
\frac{M_{0}}{\sqrt{\theta}}=\frac{\frac{r_{0}^{2}}{\theta}(\Lambda \sqrt{\theta})}{2 \exp \left(-\frac{r_{0}^{2}}{4 \theta}\right)-1}=0.214 .
$$

The variation of this factor with respect to the horizon radius is shown in Fig. 2. At this point let us also plot $\rho \sqrt{\theta}$ from Eq. (7) for various values of $M / \sqrt{\theta}$ (Fig. 3). Similarly, Fig. 4 shows $p_{t} \sqrt{\theta}$ plotted against $r / \sqrt{\theta}$ from Eq. (9).

It is worth noting that the radius of the extremal black hole is always less than the radius of the outer horizon. The result significant: if the initial mass of the black hole is $M>M_{0}$, then it can radiate until the value $M_{0}$ is reached. It follows that evaporation of the black hole may indeed be occurring.

Next, consider the Hawking temperature, which is given by

$$
\begin{aligned}
T_{H}=\frac{1}{4 \pi}\left(\frac{d g_{t t}}{d r}\right) & \left.\sqrt{-g^{t t} g^{r r}}\right|_{r=r_{h}} \\
= & -\frac{r_{h}}{4 \pi}\left[2 \Lambda+\frac{M}{\theta} \exp \left(-\frac{r_{h}^{2}}{4 \theta}\right)\right] .
\end{aligned}
$$

The plot, Fig. 5, is obtained from

$$
T_{H}=-\frac{r_{h} / \sqrt{\theta}}{4 \pi}\left[2 \Lambda \sqrt{\theta}+\frac{M}{\sqrt{\theta}} \exp \left(-\frac{r_{h}^{2}}{4 \theta}\right)\right] .
$$

Eq. (19) and Fig. 5 show that the noncommutative geometry leads to the minimal horizon radius $r_{0}$, since $T_{H}$ cannot be negative. This is exactly where $\frac{d g_{t t}}{d r}=0$ in Eq. 


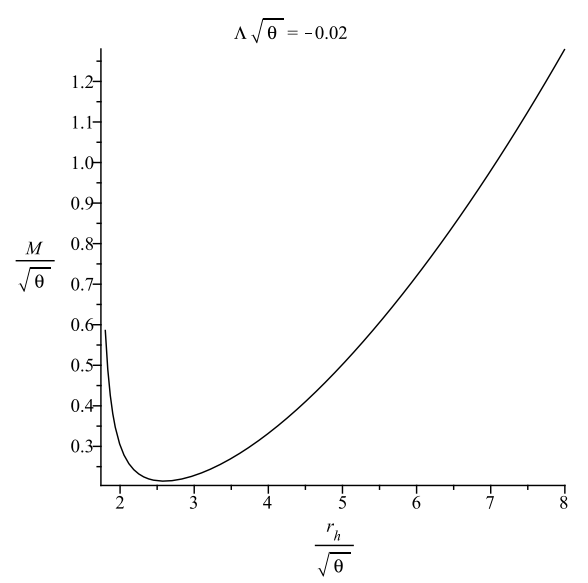

FIG. 2: The variation of mass $\frac{M}{\sqrt{\theta}}$ with respect to horizon radius $\frac{r_{h}}{\sqrt{\theta}}$.

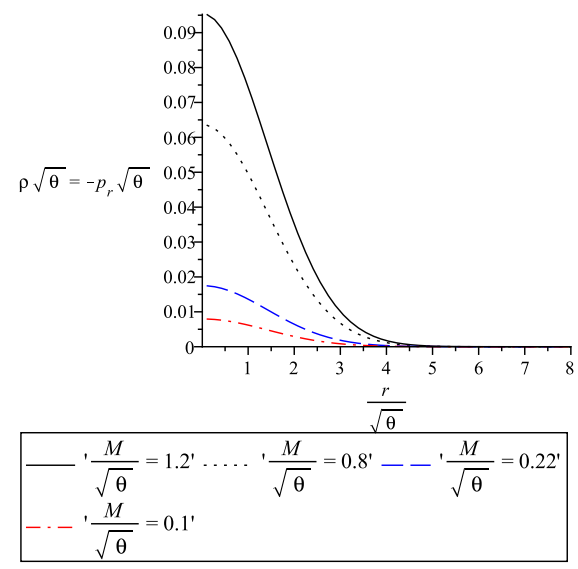

FIG. 3: Plot for $\rho \sqrt{\theta}$ vs. $\frac{r}{\sqrt{\theta}}$ for different values of $\frac{M}{\sqrt{\theta}}$. Representation: the solid curve for $M=1.2 \sqrt{\theta}$, dotted curve for $M=0.8 \sqrt{\theta}$, dashed curve for $M=0.214 \sqrt{\theta}$, and chain curve for $M=0.1 \sqrt{\theta}$.

(10). Observe that the temperature sinks to absolute zero at $r_{0}$. For $\frac{r_{h}}{\sqrt{\theta}}>>1$, the Hawking temperature assumes the value

$$
T_{H} \approx-\frac{r_{h} \Lambda}{2 \pi}
$$

For completeness, let us also state the closely related surface gravity

$$
\kappa=\left.\frac{1}{2}\left(\frac{d g_{t t}}{d r}\right)\right|_{r=r_{h}}=-\frac{r_{h}}{2}\left[2 \Lambda+\frac{M}{\theta} \exp \left(-\frac{r_{h}^{2}}{4 \theta}\right)\right],
$$

as well as the Bekenstein-Hawking entropy $(\mathrm{S})$ of the black hole. It is twice the perimeter $L$ of the event horizon:

$$
S=2 L=4 \pi r_{h}
$$

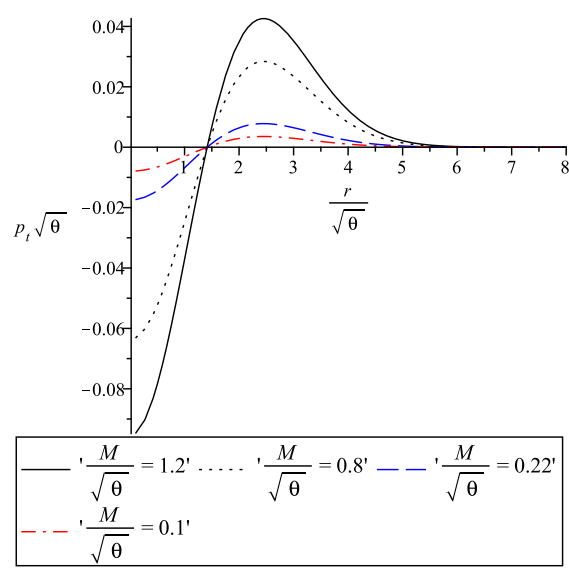

FIG. 4: Plot for $p_{t} \sqrt{\theta}$ vs. $\frac{r}{\sqrt{\theta}}$ for different values of $\frac{M}{\sqrt{\theta}}$. Representation: the solid curve for $M=1.2 \sqrt{\theta}$, dotted curve for $M=0.8 \sqrt{\theta}$, dashed curve for $M=0.214 \sqrt{\theta}$, and chain curve for $M=0.1 \sqrt{\theta}$. Note that up to the degenerate horizon $r_{0} \approx 2.59 \sqrt{\theta}$ for an extremal black hole, $p_{t}$ assumes negative values and beyond that positive values. For large values of $r$, i.e., for $\frac{r}{\sqrt{\theta}}>>1$, it dies out.

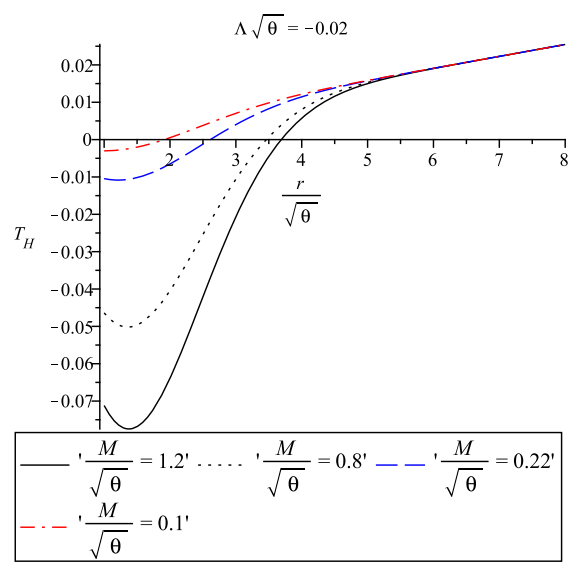

FIG. 5: Plot for Hawking temperature vs. $\frac{r}{\sqrt{\theta}}$ for different values of $\frac{M}{\sqrt{\theta}}$. Representation: the solid curve for $M=1.2 \sqrt{\theta}$, dotted curve for $M=0.8 \sqrt{\theta}$, dashed curve for $M=0.214 \sqrt{\theta}$, and chain curve for $M=0.1 \sqrt{\theta}$.

As a final comment, the noncommutative geometry inspired BTZ black hole is stable if the heat capacity $C$ is positive [22], where

$$
C=\frac{\partial M\left(r_{h}\right)}{\partial T\left(r_{h}\right)}=\frac{\partial M\left(r_{h}\right)}{\partial r_{h}} \frac{1}{\frac{\partial T\left(r_{h}\right)}{\partial r_{h}}} .
$$

The nature of the heat capacity is shown in Fig. 6 . The plot shows that $C$ vanishes at the extremal event horizon $r_{0}$ and becomes negative for $\frac{r_{h}}{\sqrt{\theta}}<\frac{r_{0}}{\sqrt{\theta}}$, just as in the case of the Hawking temperature. So this region is definitely unphysical. On the other hand, for $\frac{r_{h}}{\sqrt{\theta}}>\frac{r_{0}}{\sqrt{\theta}}$, $C$ is positive, which implies that the BTZ black hole is 


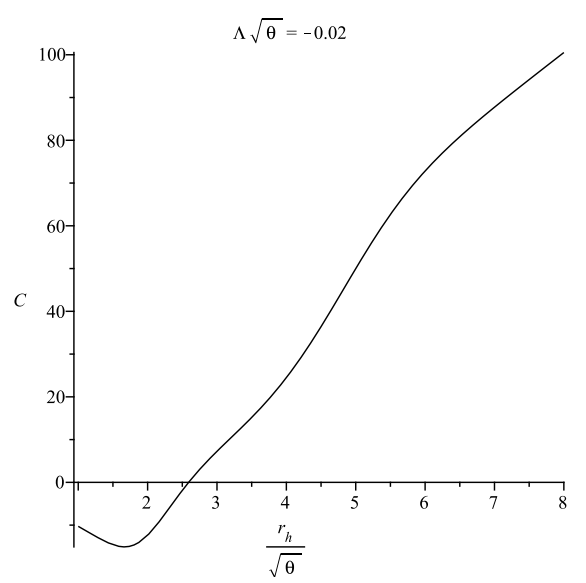

FIG. 6: Plot for C vs. $\frac{r_{h}}{\sqrt{\theta}}$.

stable.

At this point we would like to comment on how the physical quantities such as temperature, entropy, etc., could be affected by the noncommutativity for small $\theta$. We calculate the different physical quantities of the standard BTZ black hole plus perturbative terms. The BTZ black hole has the horizon located at

$$
r_{h}=\sqrt{M} l,
$$

where $\Lambda=-\frac{1}{l^{2}}$ to emphasize that $\Lambda$ is negative.
Equation (14) can be solved by iteration. The result is

$$
r_{h}=\sqrt{M} l\left[1-2 \exp \left(-\frac{M l^{2}}{4 \theta}\right)\right]^{\frac{1}{2}} .
$$

For small $\theta$ and $\frac{r_{h}}{\sqrt{\theta}}>>1$, the above equation can be written as

$$
r_{h} \approx \sqrt{M} l\left[1-\exp \left(-\frac{M l^{2}}{4 \theta}\right)\right] .
$$

Here the first term is the BTZ black hole horizon radius, while the second term is the $\theta$ correction.

It now becomes apparent that the effect of noncommutativity is small, as expected, because spacetime should be a smooth classical manifold at large distances.

The next step is to find the $\theta$ corrections of the Bekenstein-Hawking entropy (S) of the BTZ black hole:

$$
S=4 \pi r_{h} \approx 4 \pi \sqrt{M} l-4 \pi \sqrt{M} l \exp \left(-\frac{M l^{2}}{4 \theta}\right) .
$$

Here the first term is the Bekenstein-Hawking entropy (S) of the BTZ black hole, while the second term is the $\theta$ correction.

Our final task is to find the $\theta$ corrections for the Hawking temperature and surface gravity of the BTZ black hole.

From eq. (18), we have,

$$
T_{H}=-\frac{1}{4 \pi}\left[\sqrt{M} l\left\{1-\exp \left(-\frac{M l^{2}}{4 \theta}\right)\right\}\right]\left[-\frac{2}{l^{2}}+\frac{M}{\theta} \exp \left\{-\frac{M l^{2}}{4 \theta}\left[1-2 \exp \left(-\frac{M l^{2}}{4 \theta}\right)\right]\right\}\right]
$$

Keeping the first order of $\exp \left(-\frac{M l^{2}}{4 \theta}\right)$, the above expres- $\quad$ sion yields

$$
T_{H} \approx \frac{\sqrt{M}}{2 \pi l}-\left[\frac{M \sqrt{M} l}{4 \pi \theta} \exp \left\{-\frac{M l^{2}}{4 \theta}\right\}+\frac{\sqrt{M}}{2 \pi l} \exp \left\{-\frac{M l^{2}}{4 \theta}\right\}\right] .
$$

Note that the first term is the Hawking temperature of the BTZ black hole, while the second term is the $\theta$ cor- rection.

The correction term of surface gravity is given by

$$
\kappa \approx \frac{\sqrt{M}}{l}-\left[\frac{M \sqrt{M} l}{2 \theta} \exp \left\{-\frac{M l^{2}}{4 \theta}\right\}+\frac{\sqrt{M}}{l} \exp \left\{-\frac{M l^{2}}{4 \theta}\right\}\right]
$$

\section{THE GEODESICS}

From the geodesics equation

$$
\frac{d^{2} x^{\mu}}{d \tau^{2}}+\Gamma_{\nu \lambda}^{\mu} \frac{d x^{\nu}}{d \tau} \frac{d x^{\lambda}}{d \tau}=0
$$

we obtain [29]

$$
\frac{1}{f(r)}\left(\frac{d r}{d \tau}\right)^{2}=\frac{E^{2}}{f(r)}-\frac{p^{2}}{r^{2}}+L
$$




$$
r^{2}\left(\frac{d \phi}{d \tau}\right)=p
$$

and

$$
\frac{d t}{d \tau}=\frac{E}{f(r)},
$$

where $f(r)=-M+2 M \exp \left(-\frac{r^{2}}{4 \theta}\right)-\Lambda r^{2}$ and the constants $E$ and $p$ are identified as the energy per unit mass and angular momentum, respectively. Here $\tau$ is the affine parameter and $L$ is the Lagrangian having values 0 and -1 , respectively, for massless and massive particles.

From the geodesic Eq. (31) we can write

$$
\frac{1}{2}\left(\frac{d r}{d \tau}\right)^{2}=\frac{1}{2}\left[E^{2}+f(r)\left(L-\frac{p^{2}}{r^{2}}\right)\right] .
$$

Now, comparing Eq. (34) with $\frac{\dot{r}^{2}}{2}+V_{\text {eff }}=0$, the effective potential can be written

$$
V_{\text {eff }}=-\frac{1}{2}\left[E^{2}+f(r)\left(L-\frac{p^{2}}{r^{2}}\right)\right] .
$$

\section{A. Null geodesics}

For massless particles, i.e., for photons, we have $L=0$, and the corresponding effective potential is

$$
V_{e f f}=-\frac{E^{2}}{2}+\frac{p^{2}}{r^{2}}\left[-M+2 M \exp \left(-\frac{r^{2}}{4 \theta}\right)-\Lambda r^{2}\right] .
$$

As $r \rightarrow 0$, the effective potential $V_{\text {eff }}(r)$ becomes very large, but it approaches $-\frac{E^{2}}{2}-\Lambda p^{2}$ as $r \rightarrow \infty$. At the horizons, $V_{e f f}=-\frac{E^{2}}{2}$.

The shape of $V_{\text {eff }}(r)$, shown in Fig. 7, indicates that a photon will fall into a black hole [29]. Taking various values for the masses does not alter the nature of the geodesics.

\section{B. Time-like geodesics}

For massive particles, $L=-1$, and the corresponding effective potential is

$$
\begin{aligned}
& V_{\text {eff }}=-\frac{E^{2}}{2} \\
& +\left(1+\frac{p^{2}}{r^{2}}\right)\left[-M+2 M \exp \left(-\frac{r^{2}}{4 \theta}\right)-\Lambda r^{2}\right],
\end{aligned}
$$

shown in Fig. 8. The effective potential becomes very large as $r \rightarrow 0$, as well as when $r \rightarrow \infty$. At the minimal horizon $r_{0}$, it assumes the constant value $V_{\text {eff }}=-\frac{E^{2}}{2}$, while Fig. 9 shows that the roots of the $V_{e f f}$ coincide with the horizons. Also, the shape of the effective potential indicates that the particle can move only inside the

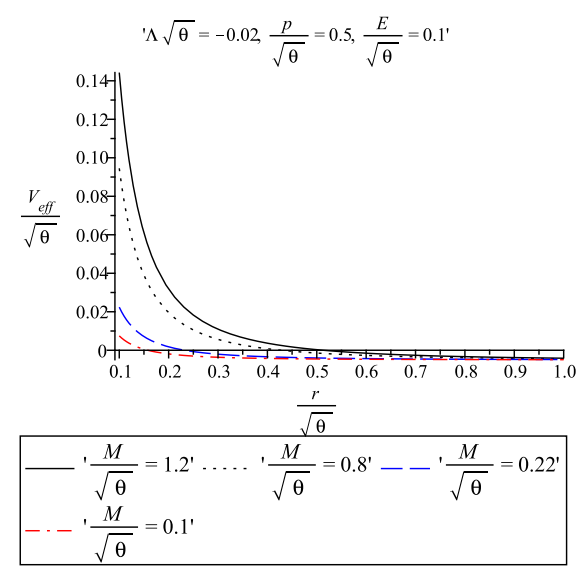

FIG. 7: Plot for $V_{e f f} \sqrt{\theta}$ vs. $\frac{r}{\sqrt{\theta}}$ for different values of $\frac{M}{\sqrt{\theta}}$. Representation: the solid curve for $M=1.2 \sqrt{\theta}$, dotted curve for $M=0.8 \sqrt{\theta}$, dashed curve for $M=0.214 \sqrt{\theta}$, and chain curve for $M=0.1 \sqrt{\theta}$. The shape of the $V_{\text {eff }} \sqrt{\theta}$ indicates that a photon will fall into black hole.

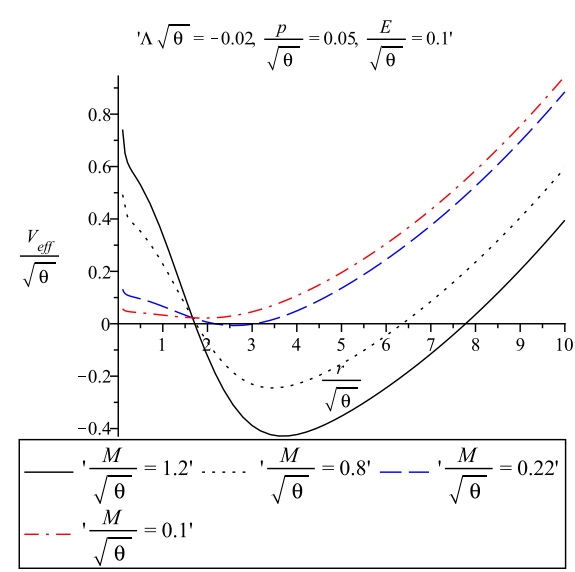

FIG. 8: Plot for $V_{e f f} \sqrt{\theta}$ vs. $\frac{r}{\sqrt{\theta}}$ for different values of $\frac{M}{\sqrt{\theta}}$. Representation: the solid curve for $M=1.2 \sqrt{\theta}$, dotted curve for $M=0.8 \sqrt{\theta}$, dashed curve for $M=0.214 \sqrt{\theta}$, and chain curve for $M=0.1 \sqrt{\theta}$. The effective potential has a minimum between two horizons, i.e., stable circular orbits do exist.

black hole. Since the effective potential assumes negative values between the horizons, the particle is confined to the region between the two horizons, and, as a result, cannot hit the singularity. Finally, observe that the minimum of $V_{\text {eff }}(r)$ occurs between the horizons, so that stable circular orbits are going to exist.

\section{TEST PARTICLES}

Let us consider a test particle having mass $m_{0}$ and moving in the gravitational field of the BTZ black hole inspired by noncommutative geometry and described by the metric ansatz (11). The Hamilton-Jacobi [H-J] equa- 


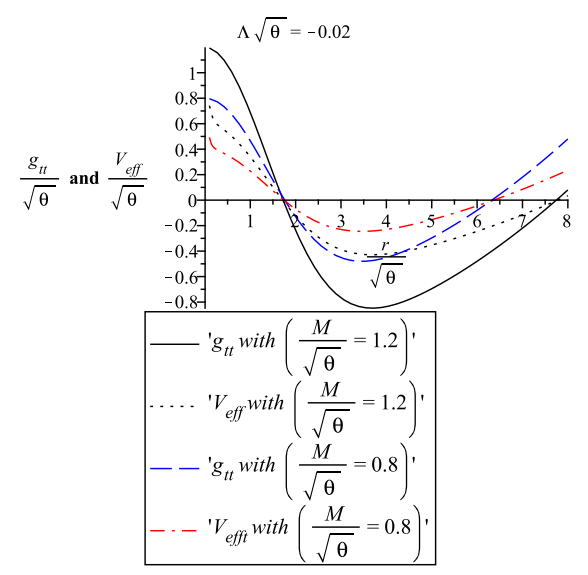

FIG. 9: Plot for $V_{e f f} \sqrt{\theta}$ vs. $\frac{r}{\sqrt{\theta}}$ for different values of $\frac{M}{\sqrt{\theta}}$. Representation: the solid curve for $M=0.8 \sqrt{\theta}$, dashed curve for $M=0.214 \sqrt{\theta}$, and chain curve for $M=0.1 \sqrt{\theta}$. The effective potential has a minimum between two horizons, i.e., stable circular orbits do exist.

tion for the test particle is 30,31$]$

$$
-\frac{1}{f}\left(\frac{\partial S}{\partial t}\right)^{2}+f\left(\frac{\partial S}{\partial r}\right)^{2}+\frac{1}{r^{2}}\left(\frac{\partial S}{\partial \phi}\right)^{2}+m_{0}^{2}=0 .
$$

As there is no explicit dependence on $t$ and $\phi$, let us choose the $H-J$ function $S$ as $[30,31]$

$$
S(t, r, \theta, \phi)=-E . t+S_{1}(r)+p . \phi,
$$

where $E$ and $p$ are identified as the energy and angular momentum of the particle.

The radial velocity of the particle is given by

$$
\frac{d r}{d t}=\frac{f^{\frac{3}{2}}}{E} \sqrt{\left[\frac{E^{2}}{f}-m_{0}^{2}-\frac{p^{2}}{r^{2}}\right]} .
$$

For detailed calculations, see Refs. [30, 31].

The turning points of the trajectory are determined from $\frac{d r}{d t}=0$ and, as a consequence, the potential curve is given by

$$
V(r) \equiv \frac{E}{m_{0}}=\sqrt{f}\left(1+\frac{p^{2}}{m_{0}^{2} r^{2}}\right)^{1 / 2} .
$$

The extremals of the potential curve are the solutions of the equation $\frac{d V}{d r}=0$ and are found to be

$$
\begin{aligned}
\frac{d V}{d r} & =-\frac{2 p^{2}}{m_{0} r^{3}}\left[-M+2 M \exp \left(-\frac{r^{2}}{4 \theta}\right)-\Lambda r^{2}\right] \\
& +\left(1+\frac{p^{2}}{m_{0} r^{2}}\right)\left[-\frac{M r}{\theta} \exp \left(-\frac{r^{2}}{4 \theta}\right)-2 \Lambda r\right]=0 .
\end{aligned}
$$

While difficult to tell from the equation, the plot of $\frac{d V}{d r}$, given in Fig. 10, shows that real positive solutions exist

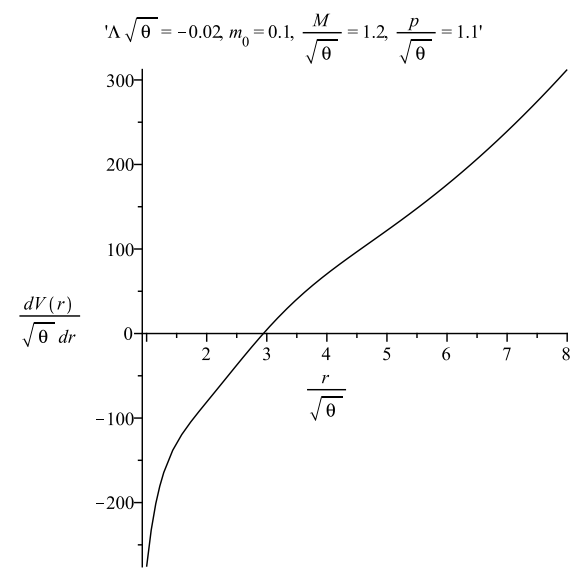

FIG. 10: Plot for $\frac{d V}{d r} \sqrt{\theta}$ vs. $\frac{r}{\sqrt{\theta}}$. Note that $\frac{d V}{d r} \sqrt{\theta}$ is zero for certain value of $\frac{r}{\sqrt{\theta}}$. This implies that $V(r)$ has at least one extremal.

wherever $\frac{1}{\sqrt{\theta}} \frac{d V}{d r}$ cuts the $\frac{r}{\sqrt{\theta}}$-axis. Hence bound orbits for the test particles exist. In other words, the test particles can be trapped by BTZ black holes inspired by noncommutative geometry.

\section{CONCLUSION}

This paper investigates the properties of a BTZ black hole constructed from the exact solution of the Einstein field equations in a $(2+1)$-dimensional anti-de Sitter spacetime in the context of noncommutative geometry.

It was found that a BTZ black hole has two horizons, no horizons or a single horizon $r=r_{0}$ corresponding to a minimal mass $M=M_{0}$. In this connection we note the comments by Mazharimousavi et al. 17]: "It is well-known that unlike its chargeless version the charged Banados-Teitelboim-Zanelli (BTZ) black hole solution in $(2+1)$-dimensional spacetime is singular". Thus they construct a charged, regular extension of the BTZ black hole solution by employing nonlinear Born-Infeld electrodynamics, supplemented with the Hoffmann term and gluing different spacetimes. However, our observation is that even the noncommutative geometry inspired BTZ black hole is not free from any singularity.

Beside this, in the present paper we continue our investigation with a discussion of Hawking temperature, entropy and heat capacity. We observe that the noncommutativity leads to the same minimal radius $r_{0}$ at which the black hole cools down to absolute zero. A discussion of the geodesic structure leads to the effective potential for both massless and massive particles. It is shown that photons will fall into the black hole, while massive particles are trapped between the two horizons. The use of the Hamilton-Jacobi equation confirms that bound orbits are possible for test particles. 


\section{Acknowledgments}

FR and SR are thankful to the authority of InterUniversity Centre for Astronomy and Astrophysics, Pune, India for providing them Visiting Associateship under which a part of this work was carried out. FR is also thankful UGC, Govt. of India under research award scheme, for providing financial support. We are very grateful to an anonymous referee for his/her insightful comments that have led to significant improvements, particularly on the interpretational aspects.
[1] M. Bañados, C. Teitelboim and J. Zanelli, Phys. Rev. Lett. 691849 (1992).

[2] A. Smailagic and E. Spalluci, J. Phys. A 36, L467 (2003).

[3] P. Nicolini, A. Smailagic, and E. Spalluci, Phys. Lett. B 632, 547 (2006).

[4] E. Spallucci, A. Smailagic, and P. Nicolini, Phys. Lett. B 670, 449 (2009).

[5] P. Nicolini and E. Spalluci, Class. Quant. Grav. 27, 015010 (2010).

[6] R. Garattini and F.R.S. Lobo, Phys. Lett. B 71, 146 (2009).

[7] F.S.N. Lobo and R. Garattini, arXiv: 1004.2520.

[8] F. Rahaman, P.K.F. Kuhfittig, K. Chakraborty, A.A. Usmani and S. Ray, Gen. Rel. Grav. 44, 905 (2012).

[9] P.K.F. Kuhfittig, AHEP 2012, 462493 (2012).

[10] F. Rahaman, P.K.F. Kuhfittig, S. Ray and S. Islam, Phys. Rev. D 86, 106010 (2012).

[11] I. Radinschi, F. Rahaman and U.F. Mondal, Int. J. Theor. Phys. 52, 96 (2013).

[12] S. Fernanado, Gen. Relativ. Gravit. 36, 71 (2004).

[13] J. Zanelli, J. Phys.: Conference Series 222, 012008 (2010).

[14] S. Nam and J.-D. Park, Class. Quant. Gravit. 26, 145015 (2009).

[15] F. Rahaman, A.A. Usmani, S. Ray and S. Islam, Phys.
Lett. B 717, 1 (2012).

[16] S.G. Ghosh, arXiv:1109.3263v2 [gr-qc].

[17] S.H. Mazharimousavi, M. Halilsoy and T. Tahamtan, Phys. Lett. A 376, 893 (2012).

[18] J.C. Lpez-Domnguez, O. Obregn, M. Sabido and C. Ramrez. Phys. Rev. D 74, 084024 (2006)

[19] O. Obregn, M. Sabido and E. Mena. Mod. Phys. Lett. A 24, 1907(2009)

[20] M. Rinaldi, Class. Quant. Gravit. 28, 105022 (2011).

[21] Y.S. Myung and M. Yoon, arXiv: 0810.0078.

[22] J. Liang and B. Liu, EPL 100, 30001 (2012).

[23] J.M. Tejeiro and A. Larranaga, Pramana - J. Phys. 78, 1 (2012).

[24] A. Larranaga and J.M. Tejeiro, Abra. Zel. J., 4, 28 (2011).

[25] J.J. Blome and W Priester, Naturwissen. 71, 528 (1984).

[26] P.C.W. Davies, Phys. Rev. D 30, 737 (1984).

[27] C. Hogan, Nat. 310, 365 (1984).

[28] N. Kaiser and A. Stebbins, Nat. 310, 391 (1984).

[29] S. Fernando, D. Krug, and C. Curry, Gen. Rel. Grav. 35, 1243 (2003).

[30] F. Rahaman and S. Chakraborty, Pramana - J. Phys. 51, 689 (1998).

[31] F. Rahaman, Int. J. Mod. Phys. D 9, 627 (2000). 